\title{
Adaptive fuzzy sliding mode control for gantry crane as varying rope length
}

\author{
TRINH LUONG MIEN \\ University of Transport and Communications \\ Falculty of Electrical and Electronic Engineering \\ Hanoi, Vietnam \\ mientl@utc.edu.vn
}

\begin{abstract}
Gantry crane is used quite commonly in hazardous areas, which increasingly requires strict conrol of the gantry crane operation process to improve efficiency and ensure safe gantry crane opeartion. Automated the gantry crane operating process is being applied pupular currently. Gantry crane is often affected by large noise, having the varying- model parameters, so that proposed a apdaptive fuzzy combining sliding mode controller for the gantry crane in this article. This control method derived from combining the sliding surfaces of three subsystem of the gantry crane (trolley position, rope length, anti-swing) to draw out two system sliding surfaces: the trolley positon with the anti-swing and the rope length and the anti-swing. On the based of the sliding mode control principle,drawn out the equivalent controller and the switching controller for gantry crane. But due to the uncertain parameters - nonlinear model of gantry crane with the bound disturbances, combining the fuzzy approximate method, defined the fuzzy controller (used to minic the equivalent controller) and the compensation controller for the difference between the equivalent controller and the fuzzy controller (used as the switching controller) for two system control inputs: trolley position and rope length The adaptive control laws for these controllers were deduced from Lyapunov's stable criteria to asymptotically stabilize the sliding surfaces. Simulation results demonstrated the feasibility of the suggested method through grantry crane in the hazard areas.
\end{abstract}

Keyword: gantry crane, adaptive controller, adaptive fuzzy controller, sliding mode control, varying rope length

\section{INTRODUCTION}

In recent years, many modern gantry cranes have brought to the increasingly common use in the transport industry for loading and unloading of goods, in the construction industry to move materials or in the manufacturing industry to assembly of heavy equipments that can not use human power. According to the traditional operation way, as moving the trolley, the hoisting rope length is fixed. However in some cases, to reduce the transportation process time, we can operate simultaneously the gantry crane moving the trolley and lifting/lowering the payloads. This will increase the risk of unsafety for people and equipments because of oscillating of the package. Although following on experimented operators, can reduce the oscillation of the payload, but that would add reducing the efficiency of freight. Therefore, automating the gantry crane operation process to enhance accuracy simultaneously for moving the trolley and lifting/lowering payload, anti-swing angle of payload, reducing transportation time, increasing safety during crane operation, is essential and is also the most basic requirements of the gantry crane operation.

Control proplem of the grantry crane is always attractive in many science reseachers and have been published on many papers in prestige science journals. Allmost of controlllers of the grantry crane are designed based on the nonlinear model or the linearized model, and unchangeable rope length. In [1] presented an adaptive controller for a linearized crane system. In [2] considered a linearized parameter-varying model of a planar crane and proposed an observer-based control design via Lyapunov equivalence function. Paper [3] dealed wih adaptive sliding mode fuzzy control approach for a linearized two dimension overhead crane system. In [4,5,6] presented PID controller combined with fuzzy logic for a linearized crane model. These methods based on the linearized crane model may lose the sufficient accuracy of information about position and load swing, so that some uncertain factors may reduce the performance of these crane control systems.

Having some papers dealed with the crane control design based on nonlinear dynamical models. In [7] presented the adaptive fuzzy controller based on the troley position error and sway angle error. The article [8] refered to the nonlinear optimal control. The article [9] presented the neural network controller for the crane. The sliding mode control (SMC) method can be found in the articles as follows: the coupling SMC [10,11], the SMC combined with fuzzy logic [12], the adaptive SMC [13, 14], the decoupling SMC [15]. The SMC method was proceeded based on the characteristics of the crane: the trolley and the hoist lifting/lowering the payload as unchanging rope length. For instance, [10,11] defined a sliding surface coupling both subsystems, [12] split a crane system and rebuilt the system states to achieve the hierarchical SMC controllers, [13] utilized such the divisibility to design a fuzzy sliding surface for trolley subsystem. The divisibility of crane systems were also adopted for the control design in [14,15]. 
Recently, several researches focused towards improving efficiency of crane operation, rapidly quenchinh the payload swing, despite of the trolley movement and lifting/lowering the winch. In $[16,17]$ presented the antiswing control of crane with changing rope length, in which the trolley motion rapidly achieved the suppression of the load sway and the asymptotic stability of the zero solutions of the position and rope length tracking errors was proved. In $[18,19]$ dealed with the controllers having compensated friction but for simply crane model, not dealing with the bound disturbance hazard areas such as wind velocity, dynamic friction.

In this paper, the gantry crane - three degree of freedom is investigated (trolley position, rope length, and sway angle), but the number of actutors is only two (trolley and hoist motors). Based on Euler-Lagrange's energy ballance equation, a dynamic model of the gantry crane is presented in section 2. In section 3, defined sliding surfaces for three decoupling subsystem of the gantry crane and then combining each pair system sliding surfaces: trolley with sway angle; and rope length with sway angle. Based on the combining sliding surfaces, proposed a new adaptive fuzzy combining sliding mode control for the gantry crane as varying the rope length and impacted by the bound disturbance hazard areas. Here proved this proposed controller according to the asymptotic stability criteria of Lyapunov's function. Then validity of the proposed method in section 4 is illustrated through simulation results. Finally, conclusions are given in section 5.

\section{GANTRY DYNAMIC MODEL}

The gantry crane is described in Fig 1 . Movement of gantry crane is procedured by the trolley and hoist motors, corresponding to two control force $F_{x}$ and $F_{l}$. Position of the load is given in $2 D-O x z$, caused by trolley motion and hoist motion, $x(t)$ - the displacement of the trolley in the $x$-direction, $l(t)$ - the hoist rope length in the $l$-direction, $\alpha(t)$ - the sway angle of the load on $x-z$ suface, $F_{f}(x)$ - the friction force of the trolley, $F_{n}(x)$ - the bound disturbance force impacting into the trolley. We have the following assumptions: the payload and trolley are connected by a massless rigid rod throught the hoist; the trolley and the payload can be regarded as point masses; the rope elongation due to tension force is negligible.

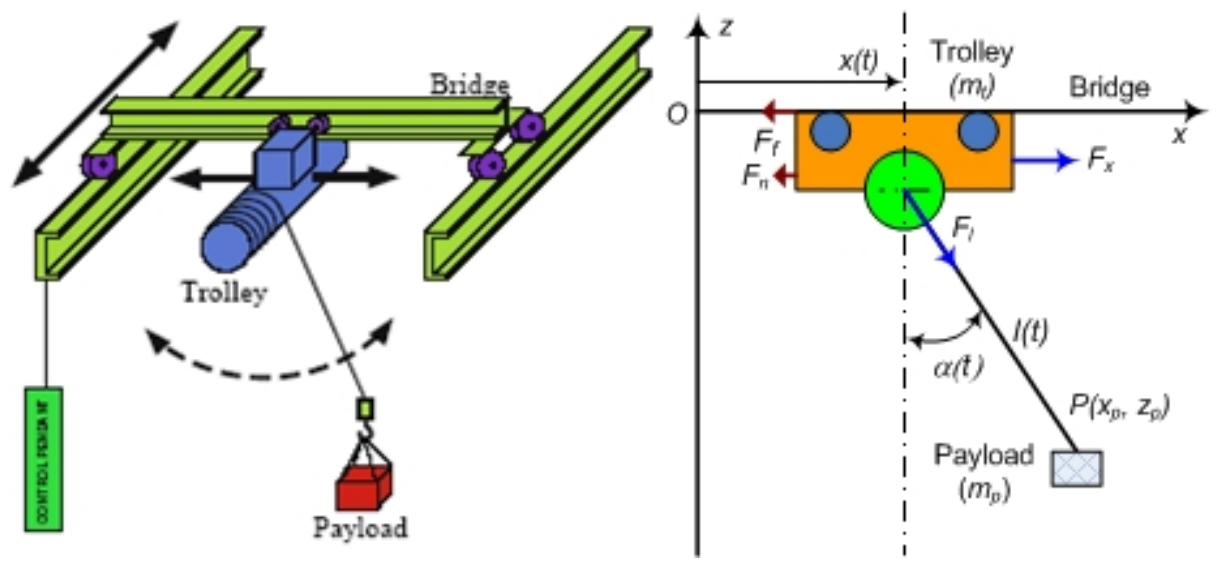

Fig 1. Constructure and diagram of the gantry crane

Let the coordinate of the payload be $\left(x_{p}, z_{p}\right), m_{p}$ is the payload mass, $m_{t}$ is the trolley mass, $g$ is the gravitational acceleration, then the system kinetic energy $T$ and the system potential energy $V$ are given as follows:

$$
\begin{gathered}
T=T_{\text {trolley }}+T_{\text {load }}=\frac{1}{2} m_{t} \dot{x}^{2}+\frac{1}{2} m_{p}\left(\dot{x}_{p}^{2}+\dot{z}_{p}^{2}\right) ; x_{p}=x+l \sin \alpha, z_{p}=-l \cos \alpha \\
V=V_{\text {trolley }}+V_{\text {load }}=-m_{p} \text { glcos } \alpha
\end{gathered}
$$

Constructing the system Lagrange $L=T-V$, we can obtain the following Lagrange equation related to the generalized coordinates $q=[x, l, \alpha]^{T}$ as:

$$
\frac{d}{d t}\left(\frac{\partial L}{\partial \dot{q}}(q, \dot{q})\right)-\frac{\partial L}{\partial q}(q, \dot{q})=\tau ; \tau=\left[F_{x}-F_{f}-F_{n}, F_{l}, 0\right]^{T}
$$

Finally, the equations of motion using the Lagrange's equation are derived as follows: 


$$
\left\{\begin{array}{l}
\left(m_{t}+m_{p}\right) \ddot{x}+m_{p}(\sin \alpha) \ddot{l}+m_{p} l(\cos \alpha) \ddot{\alpha}+2 m_{p}(\cos \alpha) \dot{l} \dot{\alpha}-m_{p} l(\sin \alpha) \dot{\alpha}^{2}=F_{x}-F_{f}-F_{n} \\
m_{p}(\sin \alpha) \ddot{x}+m_{p} \ddot{l}-m_{p} l \dot{\alpha}^{2}-m_{p} g \cos \alpha=F_{l} \\
(\cos \alpha) \ddot{x}+l \ddot{\alpha}+2 \dot{l} \dot{\alpha}+g \sin \alpha=0
\end{array}\right.
$$

Suppose that total of the friction and disturbance force $\left(-F_{f}-F_{n}\right)$ is expressed as $d(x)$, in which $|d(x)| \leq D$, the uncertainty bound $D$ is a positive constant, control input $u_{1}=F_{x}, u_{2}=F_{l}$, state variables $x_{1}=x(t), x_{2}=\dot{x}, x_{3}=l(t), x_{4}=\dot{i}, x_{5}=\alpha(t), x_{6}=\dot{\alpha}$ then above dynamic model can be transformed to the state space expression as follows:

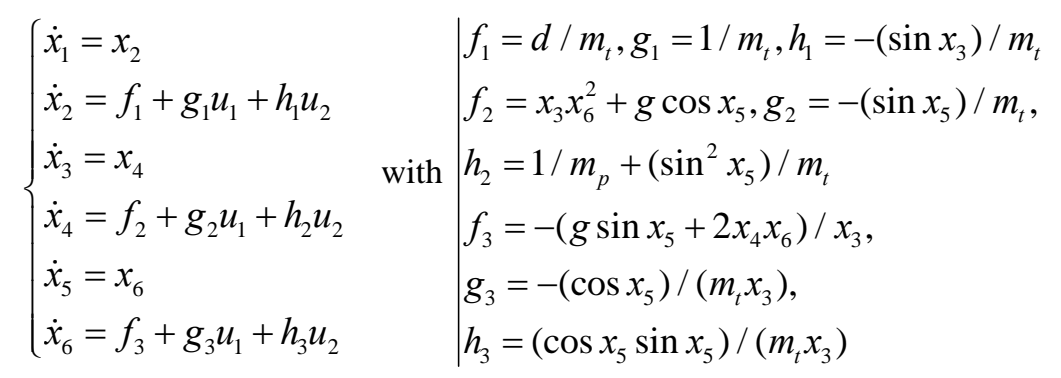

Thus, the gantry crane system is divided into three coupled subsystems: the positioning subsystem $\left(x_{1}, x_{2}\right)$, rope lenghth subsystem $\left(x_{3}, x_{4}\right)$ and anti-swing subsystem $\left(x_{5}, x_{6}\right)$. The control object of the gantry crane is to move the trolley and the varying rope lenghth to the destination and almost anti-swing of the payload when the system model exists uncertainty parameters and disturbances (for example, winds and different payloads).

\section{CONTROL DESIGN}

\section{A. Sliding mode control for gantry crane}

Assume that $x_{d}=x_{1 d}, l_{d}=x_{3 d}, x_{5 d}=\alpha_{d}$ are the input reference trajectory of trolley position, rope length and swing angle, respectively, in generally, $\alpha_{d}=0$. Define the tracking error as: $e_{1}=x_{1}-x_{d}$, $e_{3}=x_{3}-l_{d}, e_{5}=x_{5}-\alpha_{d}$ and three sliding mode functions for three subsystems as follows: $s_{x}=\dot{e}_{1}+c_{1} e_{1}$, $s_{l}=\dot{e}_{3}+c_{3} e_{3}, s_{\alpha}=\dot{e}_{5}+c_{5} e_{5}$, where $c_{1}, c_{3}, c_{5}$ are positive numbers.

In this article, a novel SMC method is applied here, by combining the sliding surfaces, i.e. using positive constants $\lambda_{1}$ and $\lambda_{2}=0$ to define a suitable pair of the system sliding surfaces as:

$$
s=\left[\begin{array}{l}
s_{1} \\
s_{2}
\end{array}\right]=\left[\begin{array}{l}
s_{x}+\lambda_{1} s_{\alpha} \\
s_{l}+\lambda_{2} s_{\alpha}
\end{array}\right]==\left[\begin{array}{l}
s_{x}+\lambda_{1} s_{\alpha} \\
s_{l}
\end{array}\right]
$$

Following on the methodology of equivalent control of variable structure control, the SMC law includes two parts: switching control law and equivalent control law. The switching control law is employed to drive the system states moving towards a specific sliding surface. The equivalent control law guarantees the system states to keep sliding on the sliding surface and converge to zero along the sliding surface. To design the combining SMC, we still adopt such the approach and define the total control law $u$ as follows:

$$
u=\left[\begin{array}{l}
u_{1} \\
u_{2}
\end{array}\right]=\left[\begin{array}{l}
u_{e q 1}+u_{s w 1} \\
u_{e q 2}+u_{s w 2}
\end{array}\right]
$$

where $u_{\text {eqi }}$ is the equivalent control and $u_{s w i}$ is the switching control $u_{i},(i=1,2)$

Letting $\dot{s}=0$, and substituting (4) into it, the equivalent control on the surface $s$ is gotten as:

$$
\begin{aligned}
& u_{e q 1}=\frac{-h_{2} f_{1}+\left(h_{1}+\lambda_{1} h_{3}\right) f_{2}-\lambda_{1} h_{2} f_{3}-c_{1} h_{2} x_{2}+c_{3}\left(h_{1}+\lambda_{1} h_{3}\right) x_{4}-\lambda_{1} c_{5} h_{2} x_{2}+h_{2}\left(\ddot{x}_{d}+c_{1} \dot{x}_{d}\right)-\left(h_{1}+\lambda_{1} h_{3}\right)\left(\ddot{l}_{d}+c_{3} \dot{l}_{d}\right)}{g_{1} h_{2}+\lambda_{1} g_{3} h_{2}-g_{2} h_{1}-\lambda_{1} g_{2} h_{3}} \\
& u_{e q 2}=\frac{-f_{2}-c_{3} x_{4}+\ddot{l}_{d}+c_{3} \dot{l}_{d}-g_{2} u_{e q 1}}{h_{2}}
\end{aligned}
$$


The equivalent control law $u_{e q 1}$ and $u_{e q 2}$ are complicated function, moreover function $f_{l}$ is uncertain, thus, the following adaptive fuzzy combining sliding mode control is proposed here.

B. Adaptive fuzzy sliding mode control

Consider two fuzzy control system $j=1,2$ for the control input $u_{f z j}$ with the $i$-th fuzzy rule as:

$$
\text { Rule } i \text { : IF } s_{j} \text { is } F_{j i} \text { THEN } u_{f j i} \text { is } \theta_{j i} \text { i } i=1,2, . . n
$$

where $F_{j i}$ are the label of the fuzzy sets characterized by the fuzzy membership functions $\mu_{F j i}(\cdot)$ and $\theta_{j i}$ are the adjustable fuzzy singletons.

The defuzzification of the output is accomplished by the method of center of gravity:

$$
u_{f z j}\left(s_{j}, \theta_{j}\right)=\frac{\sum_{i=0}^{n} \omega_{j i} \theta_{j i}}{\sum_{i=0}^{n} \omega_{j i}}=\theta_{j}^{T} \omega_{j}
$$

where $\omega_{j i}=\mu_{F j i}\left(s_{j}\right)$ is the firing weight of the $i$-th fuzzy rule;

$$
\theta_{j}=\left[\theta_{j 1}, \theta_{j 2}, \ldots, \theta_{j n}\right]^{T} ; \omega_{j}=\left[\omega_{j 1} / \sum_{i=1}^{n} \omega_{j i}, \omega_{j 2} / \sum_{i=1}^{n} \omega_{j i}, \ldots, \omega_{j n} / \sum_{i=1}^{n} \omega_{j i}\right]^{T}
$$

To estimate optimal parameters of the fuzzy system (9), use $\hat{\theta}_{j}$ instead of $\theta_{j}$, then:

$$
u_{f z j}\left(s_{j}, \hat{\theta}_{j}\right)=\hat{\theta}_{j}^{T} \omega_{j}
$$

According to fuzzy approximated theory, there exists an optimal fuzzy system $u_{f z j}^{*}(t)$ such that

$$
u_{f z j}^{*}(t)=u_{f j}^{*}\left(s_{j}, \theta_{j}^{*}\right)=\theta_{j}^{* T} \omega_{j}
$$

where the time invariant optimal parameter vector $\theta_{j}^{*}$ is defined as

$$
\theta_{j}^{*}=\arg \min _{\left|\theta_{j}\right| \leq M_{\theta_{j}}}\left\{\sup _{\left|s_{j}\right| \leq M_{s j}}\left|u_{f z j}\left(s_{j}, \theta_{j}\right)-u_{e q j}\right|\right\}, \forall t
$$

and $M_{\theta j}, M_{s j}$ are specified by the designer. The minimum approximation error is defined as

$$
\gamma_{j}(t)=u_{f z j}^{*}-u_{e q j} \quad 0 \leq\left|\gamma_{j}(t)\right| \leq \Gamma_{j}
$$

where the uncertainty bound $\hat{\Gamma}_{j}$ is a positive constant. However, this uncertainty bound can not be measured for practical application. Thus, a bound estimation $\hat{\Gamma}_{j}$ will be developed to estimate the approximation error bound $\Gamma_{j}$ of the minimum approximation error $\gamma_{j}(t)$. Define the estimation error $\tilde{\Gamma}_{j}=\Gamma_{j}-\hat{\Gamma}_{j}$, then the system control law is assumed to take the form as:

$$
u_{j}\left(s_{j}, \hat{\theta}_{j}, \hat{\Gamma}_{j}\right)=u_{f j j}\left(s_{j}, \hat{\theta}_{j}\right)+u_{c p j}\left(s_{j}, \hat{\Gamma}_{j}\right)
$$

where the fuzzy controller $u_{f z j}$ given in (9) is used to mimic the equivalent controller in (6); and the compensation controller $u_{c p j}$ given in the following is used as the switching controller in (6).

Following, we indentify the compensation controller $u_{c p j}$. Define a Lyapunov function as:

$$
V(s, \tilde{\theta}, \tilde{\Gamma})=\frac{1}{2} s^{T} s+\frac{1}{2 \eta_{1}} \tilde{\theta}^{T} \tilde{\theta}+\frac{1}{2 \eta_{2}} \tilde{\Gamma}^{T} \tilde{\Gamma}
$$

where $\tilde{\theta}=\left[\begin{array}{c}\tilde{\theta}_{1} \\ \tilde{\theta}_{2}\end{array}\right]=\left[\begin{array}{c}\theta_{1}^{*}-\hat{\theta}_{1} \\ \theta_{2}^{*}-\hat{\theta}_{2}\end{array}\right] ; \tilde{\Gamma}=\left[\begin{array}{c}\tilde{\Gamma}_{1} \\ \tilde{\Gamma}_{2}\end{array}\right]=\left[\begin{array}{c}\Gamma_{1}-\hat{\Gamma}_{1} \\ \Gamma_{2}-\hat{\Gamma}_{2}\end{array}\right]$ 
Differentiate $V$ with respect to time $t$, derive as:

$$
\dot{V}(s, \tilde{\theta}, \tilde{\Gamma})=s_{1} \dot{s}_{1}+s_{2} \dot{s}_{2}+\frac{1}{\eta_{1}}\left(\tilde{\theta}_{1}^{T} \dot{\tilde{\theta}}_{1}+\tilde{\theta}_{2}^{T} \dot{\tilde{\theta}}_{2}\right)+\frac{1}{\eta_{2}}\left(\tilde{\Gamma}_{1} \dot{\tilde{\Gamma}}_{1}+\tilde{\Gamma}_{2} \dot{\tilde{\Gamma}}_{2}\right)
$$

From (5) and (13-14), derived as:

$$
\begin{aligned}
\dot{s}_{1} & =f_{1}+\lambda_{1} f_{3}+c_{1} x_{2}+\lambda_{1} c_{2} x_{6}-\ddot{x}_{d}-c_{1} \dot{x}_{d}+\left(g_{1}+\lambda_{1} g_{3}\right)\left(u_{f z 1}+u_{c p 1}-u_{f z 1}^{*}+u_{f z 1}^{*}\right) \\
& +\left(h_{1}+\lambda_{1} h_{3}\right)\left(u_{f z 2}+u_{c p 2}-u_{f z 2}^{*}+u_{f z 2}^{*}\right) \\
\dot{s}_{1} & =f_{1}+\lambda_{1} f_{3}+c_{1} x_{2}+\lambda_{1} c_{2} x_{6}-\ddot{x}_{d}-c_{1} \dot{x}_{d}+\left(g_{1}+\lambda_{1} g_{3}\right)\left(u_{f z 1}+u_{c p 1}-u_{f z 1}^{*}+\gamma_{1}+u_{e q 1}\right)+ \\
& +\left(h_{1}+\lambda_{1} h_{3}\right)\left(u_{f z 2}+u_{c p 2}-u_{f z 2}^{*}+\gamma_{2}+u_{e q 2}\right) \\
\dot{s}_{1} & =\left(g_{1}+\lambda_{1} g_{3}\right)\left(u_{f z 1}-u_{f z 1}^{*}+u_{c p 1}+\gamma_{1}\right)+\left(h_{1}+\lambda_{1} h_{3}\right)\left(u_{f z 2}-u_{f z 2}^{*}+u_{c p 2}+\gamma_{2}\right) \\
\dot{s}_{2}= & f_{2}+\lambda_{2} x_{4}-\ddot{l}_{d}-\lambda_{2} \dot{l}_{d}+g_{2}\left(u_{f z 1}+u_{c p 1}-u_{f 21}^{*}+u_{f 21}^{*}\right)+h_{2}\left(u_{f z 2}+u_{c p 2}-u_{f z 2}^{*}+u_{f z 2}^{*}\right) \\
\dot{s}_{2}= & f_{2}+\lambda_{2} x_{4}-\ddot{l}_{d}-\lambda_{2} \dot{l}_{d}+g_{2}\left(u_{f z 1}+u_{c p 1}-u_{f z 1}^{*}+\gamma_{1}+u_{e q 1}\right)+h_{2}\left(u_{f z 2}+u_{c p 2}-u_{f z 2}^{*}+\gamma_{2}+u_{e q 2}\right) \\
\dot{s}_{2} & =g_{2}\left(u_{f z 1}-u_{f z 1}^{*}+u_{c p 1}+\gamma_{1}\right)+h_{2}\left(u_{f z 2}-u_{f z 2}^{*}+u_{c p 2}+\gamma_{2}\right)
\end{aligned}
$$

And then, combined (16) and (17), as putting $g_{13}=g_{1}+\lambda_{1} g_{3}, h_{13}=h_{1}+\lambda_{1} h_{3}$, derive as:

$$
\begin{aligned}
\dot{V}= & s_{1}\left[g_{13}\left(u_{f z 1}-u_{f z 1}^{*}+u_{c p 1}+\gamma_{1}\right)+h_{13}\left(u_{f 22}-u_{f z 2}^{*}+u_{c p 2}+\gamma_{2}\right)\right]+ \\
& +s_{2}\left[g_{2}\left(u_{f z 1}-u_{f 21}^{*}+u_{c p 1}+\gamma_{1}\right)+h_{2}\left(u_{f 22}-u_{f z 2}^{*}+u_{c p 2}+\gamma_{2}\right)\right]+\frac{1}{\eta_{1}}\left(\tilde{\theta}_{1}^{T} \dot{\tilde{\theta}}_{1}+\tilde{\theta}_{2}^{T} \dot{\tilde{\theta}}_{2}\right)+\frac{1}{\eta_{2}}\left(\tilde{\Gamma}_{1} \dot{\Gamma}_{1}+\tilde{\Gamma}_{2} \dot{\tilde{\Gamma}}_{2}\right) \\
\dot{V}= & s_{1}\left[g_{13}\left(-\tilde{\theta}_{1}^{T} \omega_{1}+u_{c p 1}+\gamma_{1}\right)+h_{13}\left(-\tilde{\theta}_{2}^{T} \omega_{2}+u_{c p 2}+\gamma_{2}\right)\right]+ \\
& +s_{2}\left[g_{2}\left(-\tilde{\theta}_{1}^{T} \omega_{1}+u_{c p 1}+\gamma_{1}\right)+h_{2}\left(-\tilde{\theta}_{2}^{T} \omega_{2}+u_{c p 2}+\gamma_{2}\right)\right]+ \\
& +\frac{1}{\eta_{1}}\left(\tilde{\theta}_{1}^{T} \dot{\tilde{\theta}}_{1}+\tilde{\theta}_{2}^{T} \dot{\tilde{\theta}}_{2}\right)+\frac{1}{\eta_{2}}\left(\Gamma_{1}-\hat{\Gamma}_{1}\right)\left(-\dot{\hat{\Gamma}}_{1}\right)+\frac{1}{\eta_{2}}\left(\Gamma_{2}-\hat{\Gamma}_{2}\right)\left(-\dot{\hat{\Gamma}}_{2}\right) \\
\dot{V}= & \frac{1}{\eta_{1}} \tilde{\theta}_{1}^{T}\left[\dot{\tilde{\theta}}_{1}-\eta_{1} \omega_{1}\left(g_{13} s_{1}+g_{2} s_{2}\right)\right]+\frac{1}{\eta_{1}} \tilde{\theta}_{2}^{T}\left[\dot{\tilde{\theta}}_{2}-\eta_{1} \omega_{2}\left(h_{13} s_{1}+h_{2} s_{2}\right)\right]+ \\
& +\left(g_{13} s_{1}+g_{2} s_{2}\right) u_{c p 1}+\frac{1}{\eta_{2}} \hat{\Gamma}_{1} \dot{\hat{\Gamma}}_{1}+\left(g_{13} s_{1}+g_{2} s_{2}\right) \gamma_{1}-\frac{1}{\eta_{2}} \Gamma_{1} \dot{\hat{\Gamma}}_{1} \\
& +\left(h_{13} s_{1}+h_{2} s_{2}\right) u_{c p 2}+\frac{1}{\eta_{2}} \hat{\Gamma}_{2} \dot{\hat{\Gamma}}_{2}+\left(h_{13} s_{1}+h_{2} s_{2}\right) \gamma_{2}-\frac{1}{\eta_{2}} \Gamma_{2} \dot{\hat{\Gamma}}_{2}
\end{aligned}
$$

According to Lyapunov's stability criteria, the necessary condition for the control system stability: $\dot{V} \leq 0$. Thereby, the compensation controller $u_{c p j}$ is given in (19) with the bound estimation law presented in (20), moreover can derive the adaptive law $\hat{\theta}_{j}$ given in (21).

$$
\begin{gathered}
u_{c p 1}=-\hat{\Gamma}_{1} \operatorname{sgn}\left(g_{13} s_{1}+g_{2} s_{2}\right) ; u_{c p 2}=-\hat{\Gamma}_{2} \operatorname{sgn}\left(h_{13} s_{1}+h_{2} s_{2}\right) \\
\dot{\hat{\Gamma}}_{1}=-\dot{\tilde{\Gamma}}_{1}=\eta_{2}\left|g_{13} s_{1}+g_{2} s_{2}\right| ; \quad \dot{\hat{\Gamma}}_{2}=-\dot{\tilde{\Gamma}}_{2}=\eta_{2}\left|h_{13} s_{1}+h_{2} s_{2}\right| \\
\dot{\hat{\theta}}_{1}=-\dot{\tilde{\theta}}_{1}=-\eta_{1} \omega_{1}\left(g_{13} s_{1}+g_{2} s_{2}\right) ; \quad \dot{\hat{\theta}}_{2}=-\dot{\tilde{\theta}}_{2}=-\eta_{1} \omega_{2}\left(h_{13} s_{1}+h_{2} s_{2}\right)
\end{gathered}
$$

Substituting (20-22) into (19), obtained as:

$$
\dot{V}=\left(g_{13} s_{1}+g_{2} s_{2}\right) \gamma_{1}-\left|g_{13} s_{1}+g_{2} s_{2}\right| \Gamma_{1}+\left(h_{13} s_{1}+h_{2} s_{2}\right) \gamma_{2}-\left|h_{13} s_{1}+h_{2} s_{2}\right| \Gamma_{2} \leq 0
$$

The negative semidefiniteness of the Lyapunov function guarantees that $s, \tilde{\theta}, \tilde{\Gamma}$ are bounded, i.e. 
function $V$ aproaches to zero ( $V \rightarrow 0$ ) as time aproaches to infinite ( $t \rightarrow \infty$ ), guaranted that:

$$
\begin{aligned}
& s \rightarrow 0 \Rightarrow s_{1} \rightarrow 0, s_{2} \rightarrow 0 \\
& \tilde{\theta} \rightarrow 0 \Rightarrow \hat{\theta}_{1} \rightarrow \theta_{1}^{*}, \hat{\theta}_{2} \rightarrow \theta_{2}^{*} \\
& \tilde{\Gamma} \rightarrow 0 \Rightarrow \hat{\Gamma}_{1} \rightarrow \Gamma_{1}, \hat{\Gamma}_{2} \rightarrow \Gamma_{2}
\end{aligned}
$$

Hence, the nonlinear gantry crane system presented in (4), the control law is designed as in (14), in which the fuzzy controller $u_{f z j}$ given in (9) with the adaptive law given in (21) and the compensation controller $u_{c p j}$ is given in (19) with the bound estimation presented in (20), then the stability of the gantry crane system can be guaranteed.

\section{SIMULATION RESULT}

In this section, we proceed the gantry crane system simulation with the suggested controlller in Matlab to validate this work. It should be emphasized that the derivation of the proposed adaptive fuzzy combining sliding mode control (AFCSMC) does not need touse the dynamic function $f_{i}(x), g_{i}(x), h_{i}(x)$ in (4), $i=1,2,3$. These dynamic functions are used only for simulations. This is the best advantage of the proposed control method than a lot of nonlinear control methods [3-15].

The control objective of the gantry crane control is to transport the payload to the required destination as fast and as accurately as possible without swings. The physical parameters of the gantry crane system in Fig 1 are determined as follows: $g=9.8\left[\mathrm{~m}^{2} / \mathrm{s}\right], m_{t}=2[\mathrm{~kg}], m_{p}=0.2[\mathrm{~kg}], l=1[\mathrm{~m}], x=1[\mathrm{~m}], c_{1}=0.5, c_{3}=0.6, c_{5}=0.8$, $\lambda_{1}=0.7, \lambda_{2}=0, \eta_{1}=50, \eta_{2}=0.001$ and the membership functions are constructed for the antecedent part; the value of the center of the triangular type membership functions are given as [-3.5 -1.50 .500 .51 .5 3.5]. These parameters are chosen trough some trials.

The block diagram of the adaptive fuzzy combining sliding mode control for the gantry crane is described in Fig 2. The AFCSMC controller consists of the sliding surfaces block given in (5), the fuzzy controller block given in (9) with the adaptive law given in (21) and the compensation controller block given in (19) with the bound estimation presented in (20).

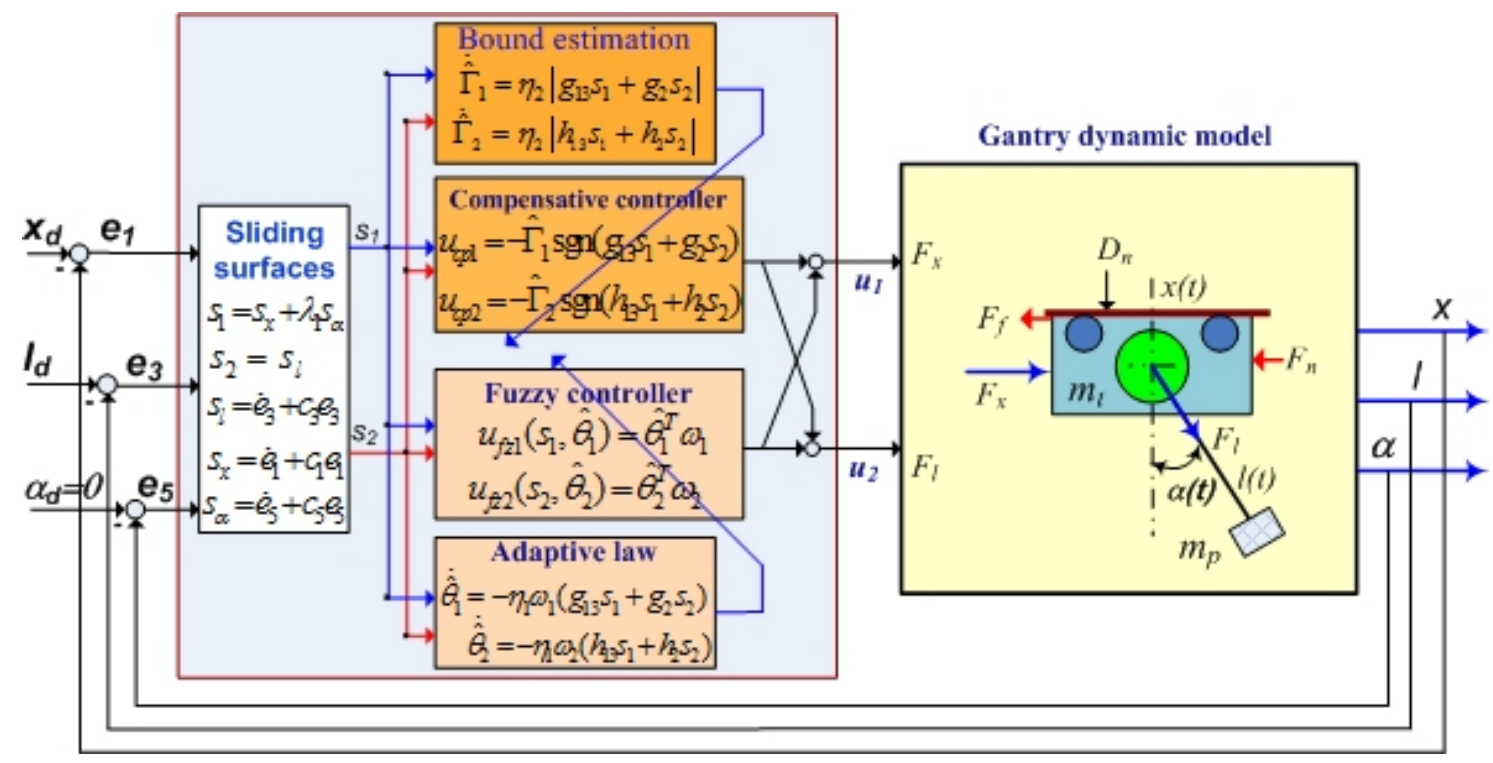

Fig 2. Proposed adaptive fuzzy combining sliding mode control for gantry crane

The simulation was carried out in three crane operating circumstances: (1) - changing the trolley position without rope length, (2) - varying the rope length without the trolley movement, (3) - changing both the trolley position and the rope length, and using the bound disturbance hazard areas (including friction force and disturbance force) describled in Fig 3. Simulation results of the AFCSMC as comparing with the PID controller $[19,20]$ were presented in Fig 3 . The simulation script was as follow: the first time $t=0$, the trolley moved to the location $0.5 \mathrm{~m}$ and lifting the payload at high $0.6 \mathrm{~m}$; then $t=10 \mathrm{sec}$, varied the rope length to lower height $0.2 \mathrm{~m}$; after finishing the additional payload process $t=20 \mathrm{sec}$, varied the rope length to lift up the payload at hight $1 \mathrm{~m}$ and, and at the same time drived the trolley to destination $0.9 \mathrm{~m}$; before the trolley stopped at target, it should be varied the rope length to lower the payload, possibly at time $t=30 \mathrm{sec}$. 
The simulation results in Fig 3 showed that as having the bound noise impacted in the gantry crane with small margin (as Fig 3), both the PID controller [19,20] and AFCSMC controller are to meet all requirements of the gantry crane operation, satisfied the payload position (trolley position, rope length) and the sway angle, but AFCSMC for better quality control: faster response time (trolley position, rope length), eliminated steady error, smaller payload oscillating amplitude $\left(<7^{\circ}\right)$ and quenched rapidly oscillating payload $(<5 \mathrm{sec})$ despite of moving simultaneously the trolley and lifting/lowering the payload.

However, when a large noise (as Fig 3), the PID controller does not meet operational requirements of the gantry crane: the trolley position was strongly oscillated, the sway angle of payload was oscillated sine sharp or with ascending amplitude $\left(>90^{\circ}\right)$, while the AFCSMC controller still allowed smoothly the gantry crane operation: responsed the trolley position was small swing around the reference trajectory but still within tolerance limit $(<2 \%)$, at the departure time $t=0.5 \mathrm{sec}$, swing angle was quite large oscillated (maximuns amplitude $\left.<15^{\circ}\right)$, then fell rapidly down the small oscillation amplitude $\left(<9^{\circ}\right)$. This proved that the proposed AFCSMC controller can be applied to operate the gantry crane in the hazardous areas.
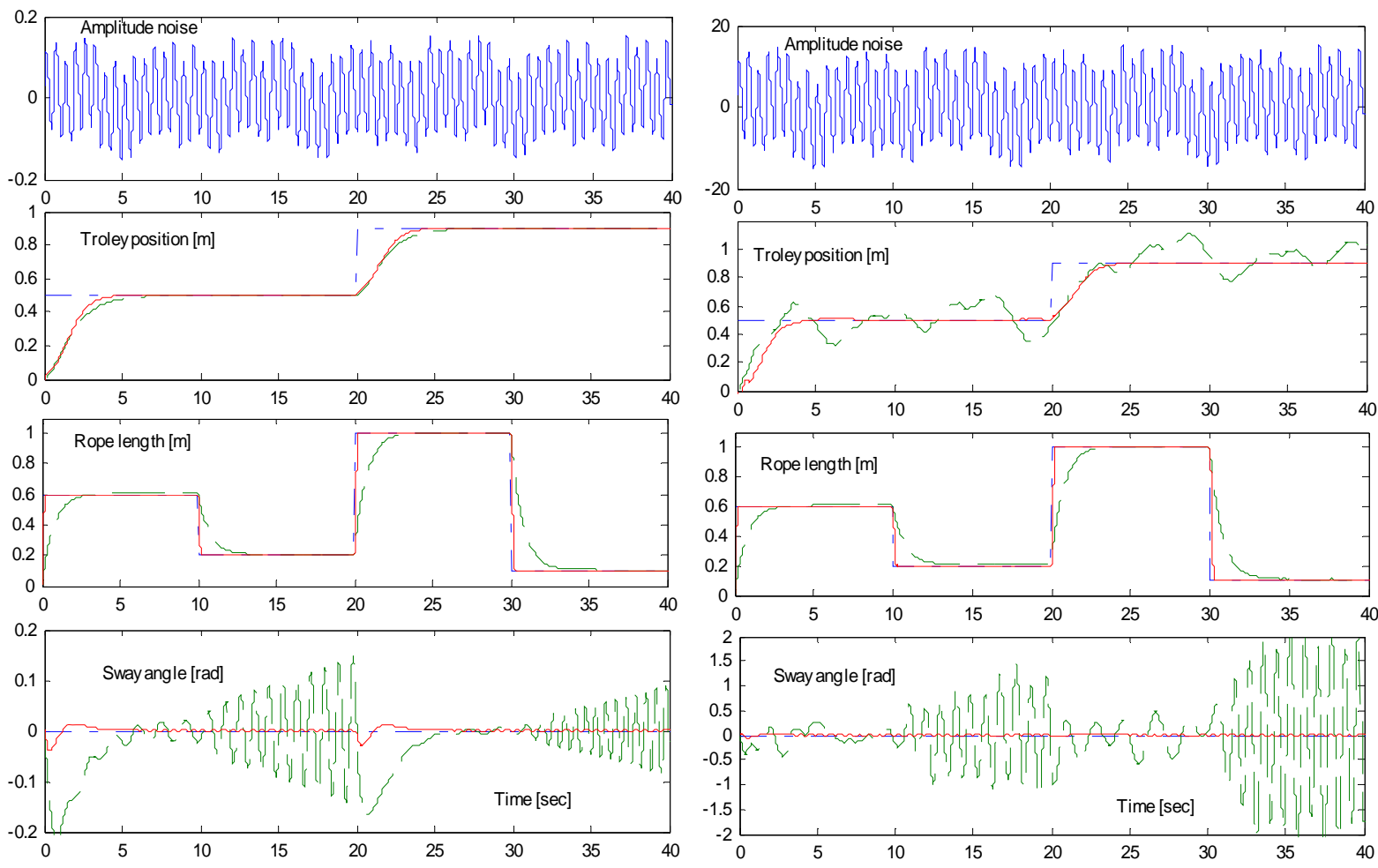

Fig 3. System performance with the AFCSMC and PID controllers, noted '-.' as reference trajectory, '--' response curves with PID, '-' response curves with AFCSMC.

\section{CONCLUSION}

Gantry crane is alway operated in hazardous areas and influenced of large disturbances, so the grantry crane mathematical model is almost not defined. This article dealed with a gantry crane model, allow simultaneously to moving the trolley and the lifting/lowering payload, from that proposed a adaptive fuzzy sliding mode control on the base of combination of the system sliding surfaces: the trolley with the swing angle and the rope length with the swing angle. The AFCSMC controller was drawn and proven according to the stability criteria of Lyapunov's function, guaranteed sufficient condition of the stability of the system states. Simulation results validated the proposed controller can applied into the transport control problem of the gantry crane system in the hazardous areas.

\section{REFERENCES}

[1] H. Butler, G. Honderd and J. Van Amerongen, Model Reference Adaptive Control of a Gantry Crane ScaleModel, IEEE Control System Magzine, vol. 11, 1991, pp. 57-62

[2] A. Giua, C. Seatzu and G. Usai, Observer-Controller Design for Cranes via Lyapunov Equivalence, Automatica, vol. 35, (1999), pp. 669-678

[3] D. T. Liu, J. Q. Yi, D. B. Zhao and W. Wang, Adaptive Sliding Mode Fuzzy Control for a Two-Dimensional Overhead Crane, Mechatronics, vol. 15, 2005, pp. 505-522

[4] Amanpreet Kaur, Priyahansha, Shashiprabha Kumari, Tanvi Singh, Position Control of Overhead Cranes Using fuzzy Controller, International Journal of Advanced Research in Electrical, Electronics and Instrumentation Engineering, 2014.

[5] A. K. Pal and R. K. Mudi, An Adaptive PD-Type FLC and Its Real Time Implementation to Overhead Crane Control, International Association of Scientific Innovation and Research, 2013. 
[6] M. Nazemizadeh, A PID Tuning Method for Tracking Control of an Underactuated GantryCrane, Universal Journal of Engineering Mechanics, 2013.

[7] Shebel Asad, Maazouz Salahat, Design of Fuzzy PD-Controlled Overhead Crane System with Anti-Swing Compensation, S. ASAD ET AL Engineering, 2011.

[8] Mahan Mahrueyan and Hamid Khaloozadeh, Designing a Nonlinear Optimal Anti-Sway Controller for Container Crane Systems, International Conference on Circuits, System and Simulation, 2011.

[9] CHEN Zhi-mei, MENG Wen-jun, ZHANG Jing-gang, Intelligent NN anti-swing control for bridge crane, J. Cent. South Univ., 2012.

[10] Shibly Ahmed AL-Samarraie, Bashar Fateh Midhat, Sliding Mode Controller Design for a Crane Container System, IJCCCE, 2014

[11] K. K. Shyu, C. L. Jen and L. J. Shang, Design of Sliding-Mode Controller for Anti-Swing Control of Overhead Cranes, Proceedings of the 31st Annual Conference of IEEE Industrial Electronics Society, vol. 1, 2005, pp. 147-152

[12] Diantong Liu, Jianqiang Yi, Dongbin Zhao, Wei Wang, Adaptive sliding mode fuzzy control for a two-dimensional overhead crane, Mechatronics 15, 2005.

[13] M. S. Park, D. Chwa and S. K. Hong, Antisway Tracking Control of Overhead Cranes With System Uncertainty and Actuator Nonlinearity Using an Adaptive Fuzzy Sliding-Mode Control, IEEE Transactions on Industrial Electronics, vol. 55, 2008, pp. 3972-3984

[14] Q. H. Ngo and K. S. Hong, Adaptive sliding mode control of container cranes, IET Control Theory and Applications, vol. 6, 2012, pp. 662-668.

[15] K. W. Choi and J. S. Lee, Sliding mode control of overhead crane, International Journal of Modelling and Simulation, vol. 31, 2011, pp. 203-209

[16] H. H. Lee, A new approach for the anti-swing control of overhead cranes with high-speed load hoisting, International Journal of Control, vol. 76, no. 15, pp. 1493-1499, 2003.

[17] Hahn Park, Dongkyoung Chwa, and Keum-Shik Hong, A Feedback Linearization Control of Container Cranes: Varying Rope Length, International journal of control automation and system, vol.5, no.4, 2007.

[18] Quang Hieu Ngoa, Trung Tinh Tranb, Keum-Shik Hongc, Anti-sway control of container cranes in the presence of friction, International Journal of Innovative Management, Information and Production, 2012.

[19] Jadranko Matu, Fetah Koloni, Friction compensation of gantry crane model based on the B-spline neural compensator, $14^{\text {th }}$ International Power Electronics and Motion Control Conference, 2010.

[20] Z.N. Masoud, A.H. Nayfeh, and N.A. Nayfeh, Sway reduction on quayside container cranes using delayed feedback controller: simulations and experiments, Journal of Vibration and Control 11, 2005.

\section{AUTHOR PROFILE}

Trinh Luong Mien obtained his PhD degree in automation and control of technological processes and manufactures at Moscow State University of Railway Engineering (MIIT) in Russia Federation in 2012.

Trinh Luong Mien is a lecturer at Faculty of Electrical and Electronic Engineering - University Transport \& Communications in Vietnam since 2004. His main research is the development of intelligent control algorithms for the technological and manufacturing processes in industry and transportation based on fuzzy logic, neuron network, adaptive \& optimal theory; study control algorithms \& guarantee safe movement of the electrical train in ATP/ATO/ATS/ATC system of the urban railway; design of supervisory control and multi-channel data collection systems. 